\title{
Quantum Dynamics of Spin Wave Propagation Through Domain Walls
}

\author{
S. Yuan ${ }^{1}$, H. De Raedt ${ }^{1}$ and S. Miyashita ${ }^{2,3}$ \\ ${ }^{1}$ Department of Applied Physics, Materials Science Center, \\ University of Groningen, Nijenborgh 4, NL-9747 AG Groningen, The Netherlands \\ ${ }^{2}$ Department of Physics, Graduate School of Science, \\ University of Tokyo, Bunkyo-ku, Tokyo 113-0033, Japan \\ ${ }^{3}$ CREST, JST, 4-1-8 Honcho Kawaguchi, Saitama, Japan
}

\begin{abstract}
Through numerical solution of the time-dependent Schrödinger equation, we demonstrate that magnetic chains with uniaxial anisotropy support stable structures, separating ferromagnetic domains of opposite magnetization. These structures, domain walls in a quantum system, are shown to remain stable if they interact with a spin wave. We find that a domain wall transmits the longitudinal component of the spin excitations only. Our results suggests that continuous, classical spin models described by LLG equation cannot be used to describe spin wave-domain wall interaction in microscopic magnetic systems.
\end{abstract}

KEYWORDS: quantum spin model, nanomagnetic, domain wall, Schrödinger equation

\section{Introduction}

Wave propagation in one-dimensional magnets through a magnetic domain wall (DW) is an interesting topic in quantum many-body physics. A DW separates two regions with opposite magnetization. The DW in mesoscopic wire can be considered to be self-assembled stable nanostructures which is treated as a kind of soliton in a continuous medium. Such structure can be created or annihilated by some external action. ${ }^{1}$ The manipulation of DW in stripes has already been proposed as a way of storing information or even performing logic functions, and to offer new types of electronics devices ${ }^{1}$ in which the DW motion carries the information along a magnetic wire of submicrometer width, with DW velocities up to thousand kilometers per second. ${ }^{2}$ Recently, a direct observation of the pendulum dynamics of a DW has been reported. ${ }^{3}$ The DW as a topological particle has a very small but finite mass of $6.6 \times 10^{-23} \mathrm{~kg} .^{3,4}$

The structure of DWs and also wave propagation in one-dimensional classical spin systems has been studied in Refs. ${ }^{5,6}$ Recently, the interaction between DWs and spin waves has attracted a lot of interest. Hertel et al showed that the DW induced phase shift of spin waves in the Landau-Lifshitz-Gilbert (LLG) model of thin, narrow strips, is a characteristic property of such systems. ${ }^{8}$ The value of the phase shift of spin waves passing through a DW was found to be proportional to the angle by which the magnetization of DW rotates in the film plane. ${ }^{8}$ This effect might be used as a concept for a new generation of nonvolatile memory storage and logical devices. ${ }^{8}$

On the other hand, recent progress in synthesizing materials containing ferromagnetic chains ${ }^{9-12}$ opens new possibilities to study the interaction between a spin wave and a DW in a microscopic spin chain. Furthermore, quantum spin models provide a playground to investigate how quantum information can be transferred in quantum spin networks. ${ }^{13-15}$ But, in contrast to the nanoscale phenomena mentioned earlier, on the atomic level, the

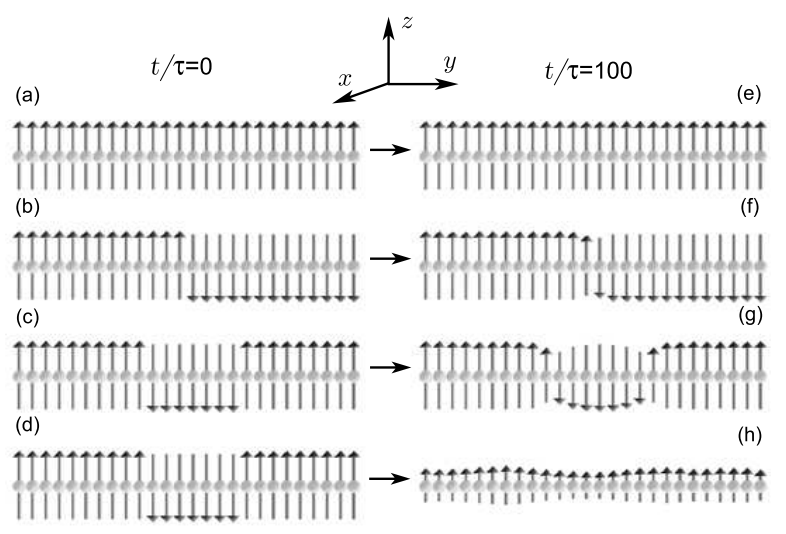

Fig. 1. Left pictures (a,b,c,d): Spin configurations at time $t / \tau=$ 0 ; Right pictures $(\mathrm{e}, \mathrm{f}, \mathrm{g})$ : Dynamically stable spin configurations for the Heisenberg-Ising model $(\lambda=2)$, at time $t / \tau=100$. (e): Ferromagnetic state of the spin chain; (f): State containing a DW at the center of the chain; $(\mathrm{g})$ : State containing two DWs. Right picture $(\mathrm{h})$ : Spin configuration at time $t / \tau=100$ for the Heisenberg model $(\lambda=1)$, illustrating the instability of the initial domain wall state $(d)$.

spin dynamics is purely quantum mechanical and in such strongly quantum fluctuating systems it must be described by the time-dependent Schrödinger equation (TDSE). Then, it is of considerable interest to compare the properties of spin wave propagation through a magnetic domain boundary in a single spin chain with the dynamics obtained in mesoscopic system, in which the magnetization is regarded as a classical, continuous variable. In the nanoscale regime, the DW is defined as the boundary of regions with opposite magnetization. On the atomic level, a DW may be defined as a structure that is dynamically stable under quantum mechanical motion, the existence of which has to be confirmed.

\section{Dynamically Stable Domain Walls}

In this paper, we study the stability of DWs and the effects of DWs on the spin wave propagation in a chain 
of $N$ sites on which we place $S=1 / 2$ spins. We solve the TDSE to compute the time-evolution of the magnetization at each lattice site. The Hamiltonian of the spin chain is given by ${ }^{16}$

$$
H=-J \sum_{n=1}^{N-1}\left(S_{n}^{x} S_{n+1}^{x}+S_{n}^{y} S_{n+1}^{y}+\lambda S_{n}^{z} S_{n+1}^{z}\right),
$$

where the exchange integrals $J>0$ and $\lambda J$ determine the strength of the interaction between the $x, y$ and $z$ components of spin $1 / 2$ operators $\mathbf{S}_{n}=\left(S_{n}^{x}, S_{n}^{y}, S_{n}^{z}\right)$. We solve the TDSE by the Chebyshev polynomial algorithm which is known to yield extremely accurate solutions of the TDSE, independent of the time step used. ${ }^{17-20} \mathrm{We}$ display the results at time intervals of $\tau=\pi / 5 \mathrm{~J}$. We present results for systems containing $N=26$ spins only. We checked that simulations for $N=20$ spins (data not shown) yield qualitatively similar results. In our numerical work, we use units such that $\hbar=1$ and $J=1$.

First, we study the stability of DWs. The left panel of Fig. 1 shows the spin configurations that we take as the initial state $(t / \tau=0)$ in the simulation. All the results shown in this Letter have been obtained using open boundary conditions. We let the system evolve in time according to the Hamiltonian Eq.(1) for a long time $(t / \tau=100)$ and find that the motion generates a dynamically stable state with DW(s) (see Fig. 1(f,g)). The DW is defined as the boundary between regions of different magnetization but it is not trivial that these boundaries exists in the presence of strong quantum fluctuations. ${ }^{7}$ Figure 1(e,f,g) shows the dynamically stable spin configurations obtained by starting from the corresponding configuration $(\mathrm{a}, \mathrm{b}, \mathrm{c})$.

Whether or not quantum fluctuations destroy the DW(s) depends on the value of the anisotropy $\lambda$. For the model Eq.(1), it is well know ${ }^{16}$ that quantum fluctuations destroy the long range order of the ground state if $-1<\lambda<1$ (XY-like) or $\lambda=-1$ (Heisenberg antiferromagnet). For $\lambda \geq 1$ (Ising-like) the ground state exhibits long range order. This property is reflected in the stability of configurations that contain one or more DWs, except for $\lambda=1$. Our numerical simulations show that configurations with a DW are dynamically stable if $\lambda>1$. For comparison, in Fig. 1 we include the case $\lambda=1$, where the initial DW structure (Fig. 1(d)) is destroyed (Fig. 1(h)). Not surprisingly, the destructive effect of quantum fluctuations can be suppressed by increasing $\lambda$. Having studied systems with different values of $\lambda$, we found that $\lambda=2$ is representative for the quantitative behavior of the anisotropic systems. Therefore, in this paper, we present results for $\lambda=2$ only. We also checked the effect of the boundary condition. We found almost the same stable DW structures in the case of periodic boundary conditions (results not shown).

\section{Spin Wave Propagation}

We use the configuration at $t / \tau=100$ as the initial configuration to study the spin wave dynamics. We generate a spin wave excitation by rotating the left most spin $\mathbf{S}_{1}$ in Fig. 1(e,f,g). For reference, we also consider the dynamics of the ferromagnet (see Fig. 1(a,e)). This (a) No DW

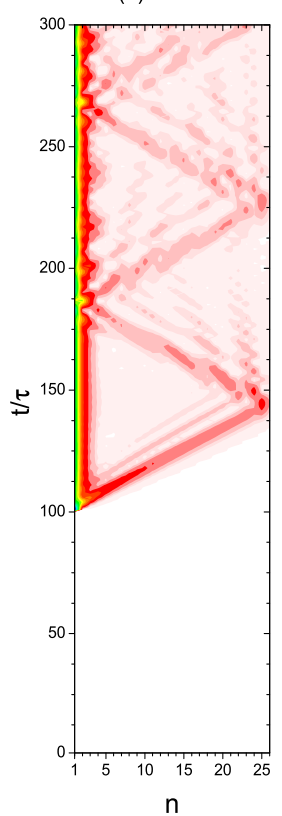

(b) One DW

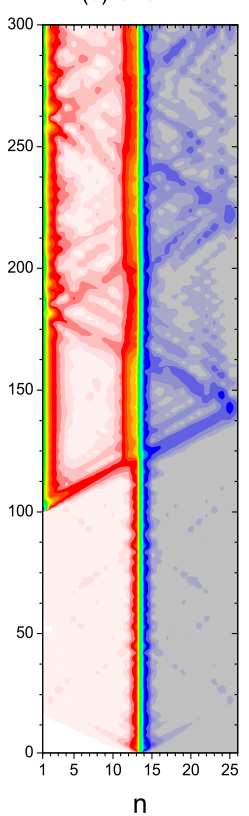

(c) Two DWs

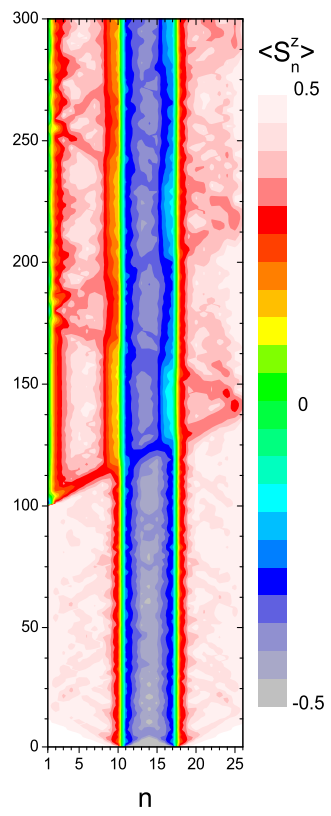

Fig. 2. (color online) Time evolution of the magnetization $\left\langle S_{n}^{z}(t)\right\rangle$ of the Ising-like spin chain with $\lambda=2$. The initial configuration $(t / \tau=0)$ of each panel $(\mathrm{a}, \mathrm{b}, \mathrm{c})$ is shown in Fig. $1(\mathrm{a}, \mathrm{b}, \mathrm{c})$, respectively. At the time $t / \tau=100$, the first $\operatorname{spin}(n=1)$ is flipped, generating a longitudinal spin wave. (a) No DW

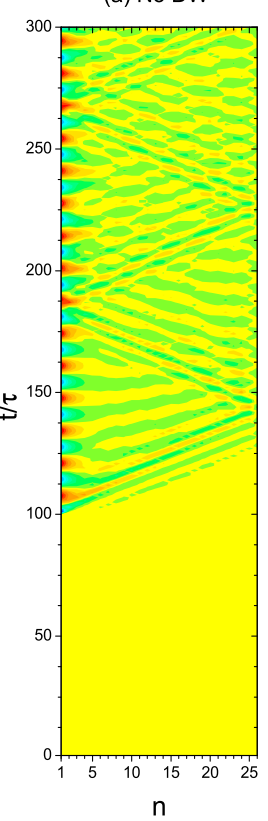

(b) One DW

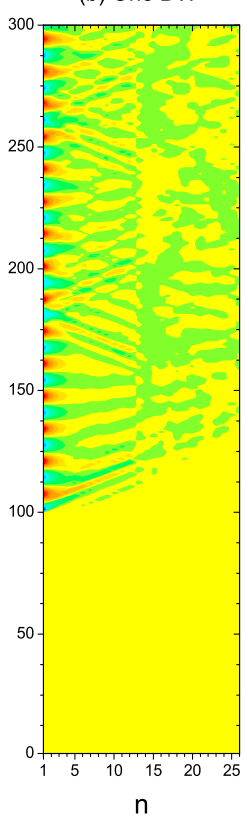

(c) Two DWs

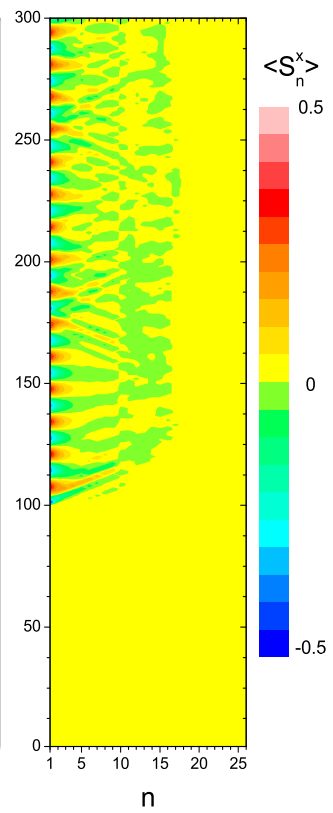

Fig. 3. (color online) Time evolution of the transverse component $\left\langle S_{n}^{x}(t)\right\rangle$ of the magnetization, for the same cases as those shown in Fig. 2 except that the first spin is rotated by $\pi / 2$ about the $y$-axis (instead of flip) at the time $t / \tau=100$.

case without DW can be analyzed analytically, so that it also gives check of precision of numerical calculation. Actually, we found very small difference between the analytical results and numerical ones.

In Fig. 2(a) we show the time evolution of $\left\{\left\langle S_{n}^{z}(t)\right\rangle\right\}$ for $n=1, \ldots N$ after flipping $\mathbf{S}_{1}$, in the case of the uniform 

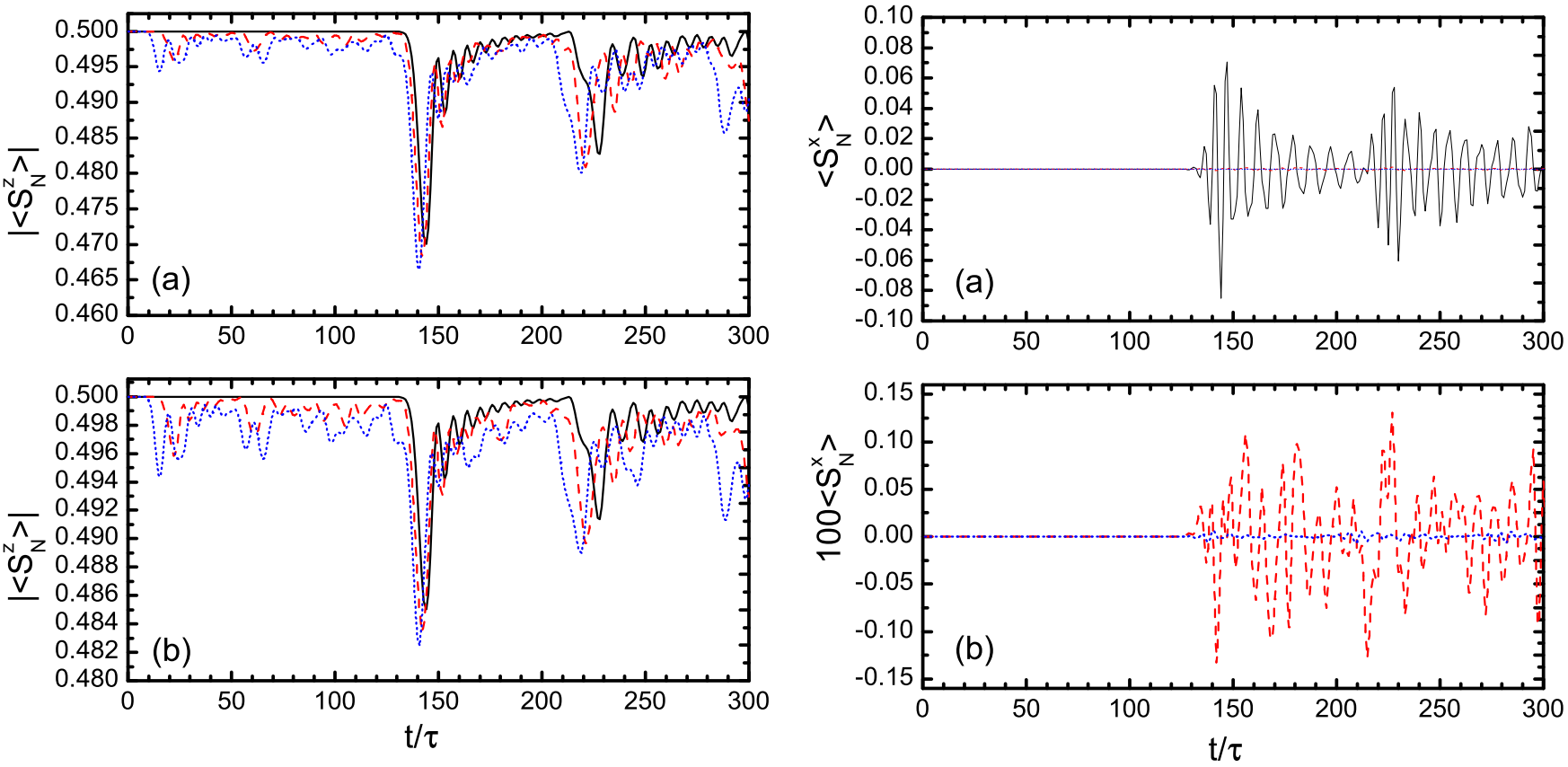

Fig. 4. (color online) Time evolution of the magnetization $\left\langle S_{N}^{z}(t)\right\rangle$. (a) spin wave generated by flipping the first spin $\mathbf{S}_{1}$; (b) spin wave generated by rotating the first spin $\mathbf{S}_{1}$ by $\pi / 2$ about the $y$-axis. Solid (black) line: No DW, corresponding to the spin configuration Fig. 1(e); Dashed (red) line: one DW, corresponding to the spin configuration Fig. 1(f). Because $\left\langle S_{N}^{z}(t)\right\rangle$ is negative in this case, we plot the absolute value to facilitate the comparison; Dotted (blue) line: two DWs, corresponding to the spin configuration Fig. 1(g). Comparison of (a) and (b) shows that the times at which the $\left\langle S_{N}^{z}(t)\right\rangle$ reaches one of the minima does not depend on method by which the spin wave is generated.

chain. The time evolution of $\left\{\left\langle S_{n}^{z}(t)\right\rangle\right\}$ for $n=1, \ldots N$ in the chain with one DW at $n=13,14$ is depicted in Fig. 2(b), and Fig. 2(c) shows the results for the chain with one DW at $n=10,11$ and another DW at $n=17,18$. Hence, we demonstrate that even in the presence of a spin wave, the DW structure remain stable. In the model Eq.(1), the magnetization in the $z$-direction is a conserved quantity. Hence, by flipping one or more spins we change the total magnetization of the initial state. The expectation value of the transverse spin components is identically zero $\left(\left\langle S_{n}^{x}(t)\right\rangle=\left\langle S_{n}^{y}(t)\right\rangle=0\right.$ for $n=1, \ldots N)$, for all $t>0$.

From Fig. 2, we can deduce how the spin wave is scattered by the DW(s). The triangular pattern in Fig. 2(a) merely results from the reflection of the spin flip excitation by the other edge of the chain. The triangular pattern is also present in Fig. 2(b), but the presence of the DW causes $\left\langle S_{n}^{z}(t)\right\rangle$ to change sign if $n>N / 2$. Fig. 2(b) also demonstrates that the DW itself is extremely robust, even in systems with one spin flipped. A similar behavior is observed for the case of two DWs (see Fig. 2(c)), indicating that the change of sign at the DW is generic.

The slope of the line in Fig. 2 from the point $(n=$ $1, t / \tau=100)$ that connects spin 1 and spin $N$ is directly related to the velocity of the excitation. We can estimate the time of the excitation to propagate from site $n$ to site $m$ by analyzing the infinitely long chain. Starting from an initial state in which we flip the spin at site $n$, the

Fig. 5. (color online) Time evolution of $\left\langle S_{N}^{x}(t)\right\rangle$ of the same system as in Fig. 3, plotted on two different scales. Solid (black) line: No DW, corresponding to the spin configuration Fig. 1(e); Dashed (red) line: One DW, corresponding to the spin configuration Fig. 1(f). Dotted (blue) line: Two DWs, corresponding to the spin configuration Fig. 1(g).

magnetization at site $m$ is given by

$$
\begin{aligned}
\left\langle S_{m}^{z}(t)\right\rangle & =\lim _{N \rightarrow \infty} \frac{1}{2}\left[1-\left|\left\langle n\left|e^{-i t H}\right| m\right\rangle\right|^{2}\right], \\
& =\frac{1}{2}\left[1-2 J_{m-n}^{2}(J t)\right]
\end{aligned}
$$

where $|m\rangle$ denotes the ferromagnet state with a flipped spin at site $m$ and $J_{m}(x)$ is the Bessel function of the first kind of order $m$. Although Eq. (3) is valid for the infinite chain only, we may expect that it provides a qualitatively correct description of the wave propagation in the finite system. Our numerical calculations (results not shown) demonstrate that for $N \geq 16$, the time for the excitation to travel from $n=1$ to $m=26$ agrees within $2 \%$ with the first minimum of Eq. (3).

Although it is clear that the longitudinal motion of the spin that results from the spin flip can easily propagate through the DW structures, quantum fluctuations reduce the amplitude of the excitation and for $t / \tau>250$ it becomes difficult to follow the excitation in Fig. 2(b,c). As mentioned earlier, we could increase $\lambda$ to reduce the quantum fluctuations but this does not change the qualitative features that we are interested in.

Next, we study the propagation of the transverse components, that is the $x$ or $y$ components of the expectation values of the spins. At $t / \tau=100$, we excite the system by rotating the first spin in Fig. $1(\mathrm{e}, \mathrm{f}, \mathrm{g})$ by $\pi / 2$ about the $y$-axis. After this rotation, the magnetization of spin $\mathbf{S}_{1}$ is parallel to the $x$-axis. Starting from this configuration, the time evolution will cause the first spin to rotate about the $z$-axis (due to the presence of the neighboring spin that is pointing in the $z$-direction). This then gener- 
ates spin waves that contain both longitudinal $\left\{\left\langle S_{n}^{z}(t)\right\rangle\right\}$ and transverse $\left(\left\{\left\langle S_{n}^{x}(t)\right\rangle\right\},\left\{\left\langle S_{n}^{y}(t)\right\rangle\right\}\right)$ components.

The space-time diagram of $\left\langle S_{n}^{z}(t)\right\rangle$ looks very similar as Fig. 2 and therefore we do not show it. Now, we investigate the propagation of the transverse spin waves by considering one of the two components (the actual choice is irrelevant). In Fig. 3, we present results for the time evolution of $\left\langle S_{n}^{x}(t)\right\rangle$ for $n=1$ to $N$. In the Isinglike Heisenberg chain without a DW, the transverse spin waves propagate in the same manner as the longitudinal waves. (compare Fig. 2(a) and Fig. 3(a)). However, from Fig. 3(b,c) it is clear that the transverse waves do not propagate through the DW structure but are reflected instead.

For a more quantitative study of the interaction of $\mathrm{DW}(\mathrm{s})$ and spin waves in quantum spin chains, we analyze in detail, the time evolution of the right-most spin. In Fig. 4 we plot $\left\langle S_{N}^{z}(t)\right\rangle$ as a function of time for the six cases depicted in Figs. 2 (Fig. 4(a)) and 3 (Fig. 4(b)).

From Fig. 4, we conclude that the propagation of longitudinal spin waves in the two cases is essentially the same, except for the amplitude. Rotating the first spin by $\pi / 2$ (instead of $\pi$ in the case of the spin flip) about the $x$ or $y$ axis generates waves of which the amplitude of the longitudinal component at the site $N$ is half of that of the spin-flip case. Using Eq. (3) and the fact that $J_{25}(x)$ has a first maximum at $x \approx 27.4$, we find that $\left\langle S_{26}^{z}(t)\right\rangle$ has a minimum at $t / \tau \approx 144$. This value is in agreement with the time at which the numerical solution for the $N=26$ chain exhibits a first dip (see black (solid) line in Fig. 4(a)). A first conclusion from this analysis is that the qualitative aspects of the interaction of the longitudinal spin wave excitation and the DW(s) does not depend on the transverse components of the spin wave.

Fig. 4 also clearly shows that the presence of a DW increases the speed at which the excitation travels through the DW. Comparing the curves for the system without DW, one DW, and two DWs, we conclude that the solid curve lags behind with respect to the dashed curve, and the dashed curve lags behind with respect to the dotted curve. Thus, the longitudinal component of the spin wave excitation is shifted forward as it passes a DW.

Fig. 5 shows the time evolution of $\left\langle S_{N}^{x}(t)\right\rangle$. In contrast to the longitudinal component (see Fig. 4), the maximum amplitude of the transverse signals strongly depend on the presence of $\mathrm{DW}(\mathrm{s})$ in the system (note the difference in scale between Fig. 5(a) and Fig. 5(b)). Thus, in the quantum system, the reflection of the transverse spin wave excitation is significantly larger than the reflection of the longitudinal component.

\section{Discussion and Summary}

Finally, we point out the difference between the continuous model for mesoscale magnetic systems and the present lattice model. In the former, DWs exist as rotation of the spins according to a soliton structure, while in the microscopic quantum system, there is no struc- ture in the transverse spin component and a DW is defined as a dynamically stable structure of the longitudinal components. We found that such DWs exists for $\lambda>1$ whereas for $\lambda \leq 1$ they are unstable. We also studied spin wave propagation and found that the longitudinal components of the spin wave speed up when they cross a DW. The transverse components of the spin wave are almost totally reflected by the DW, but this characteristic feature of the microscopic quantum chain is not found in mesoscopic magnetic system, where the transverse components crosse a DW without reflection and with a phase shift of $\pi / 2 .{ }^{8}$ It should be noted that the system described by the LLG equation is fundamentally different from the system that we consider in this Letter. The former treats the magnetic system in the mesoscopic regime as a classical, continuous medium, whereas the present study treats the magnetic system as a microscopic, quantum mechanical system. Which of these two approaches is the most suitable description obviously depends on the specific material. The change of behavior from mesoscopic to microscopic may become important as bottom-up chemical synthesis is providing new ways for further down-sizing of the magnets.

1) D.A. Allwood, G. Xiong, M.D. Cooke, C.C. Faulkner, D. Atkinson, N. Vernier and R.P. Cowburn, Science 296 (2002) 2003.

2) D. Atkinson, D.A. Allwood, G. Xiong, M.D. Cooke, C.C. Faulkner and R.P. Cowburn, Nature Materials 2 (2003) 85.

3) E. Saitoh, H. Miyajima, T. Yamaoka and G. Tatara, Nature 432 (2004) 203

4) C. Charppert and T. Devolder, Nature 432 (2004) 162.

5) K. Nakamura and T. Sasada, Phys. Lett. 48A (1974) 321.

6) K. Nakamura and T. Sasada, J. Phys. C: Solid State Phys. 11 (1978) 331.

7) H.J. Mikeska, S. Miyashita and G.H. Ristow, J. Phys.: Condens. Matter 3 (1991) 2985.

8) R. Hertel, W. Wulfhekel and J. Kirschner, Phys. Rev. Lett. 93 (2004) 257202.

9) T. Kajiwara, M. Nakano, Y. Kaneko, S. Takaishi, T. Ito, M. Yamashita, A. Igashira-Kamiyama, H. Nojiri, Y. Ono and N. Kojima, J. Am. Chem. Soc. 127 (2005) 10150.

10) M. Mito, H. Deguchi, T. Tajiri, S. Takagi, M. Yamashita and H. Miyasaka, Phys. Rev. B 72 (2005) 144421.

11) H. Kageyama, K. Yoshimura, K. Kosuge, M. Azuma, M Takano, H. Mitamura and T. Goto, J. Phys. Soc. Jpn. 66 (1997) 3996.

12) A. Maignana, C. Michel, A.C. Masset, C. Martin and B. Raveau, Eur. Phys. J. B 15 (2000) 657.

13) S. Bose, Phys. Rev. Lett. 91 (2003) 207901.

14) T.J. Osborne and N. Linden, Phys. Rev. A 69 (2004) 052315.

15) M. Christandl, N. Datta, A. Ekert and A.J. Landahl, Phys. Rev. Lett. 92 (2004) 187902.

16) D.C. Mattis, The Theory of Magnetism I, Solid State Science Series 17 (Springer, Berlin 1981).

17) H. Tal-Ezer and R. Kosloff, J. Chem. Phys. 81 (1984) 3967.

18) C.Leforestier, R.H. Bisseling, C. Cerjan, M.D. Feit, R. Friesner, A. Guldberg, A. Hammerich, G. Jolicard, W. Karrlein, H.-D. Meyer, N. Lipkin, O. Roncero and R. Kosloff, J. Comp. Phys. 94 (1991) 59.

19) T. Iitaka, S. Nomura, H. Hirayama, X. Zhao, Y. Aoyagi and T. Sugano, Phys. Rev. E56 (1997) 1222.

20) V.V. Dobrovitski and H.A. De Raedt, Phys. Rev. E67 (2003) 056702 . 